\title{
Mate availability does not influence mating strategies in males of the sexually cannibalistic spider Argiope bruennichi
}

\author{
Anna-Lena Cory ${ }^{\text {Corresp., }}{ }^{1}$, Jutta M. Schneider ${ }^{1}$ \\ 1 Institute of Zoology, Universität Hamburg, Hamburg, Germany \\ Corresponding Author: Anna-Lena Cory \\ Email address: Anna-Lena.Cory@uni-hamburg.de
}

Background. Sexual selection theory predicts that male investment in a current female should be a function of female density and male competition. While many studies have focused on male competition, the impact of female density on male mating investment has been widely neglected. Here, we aimed to close this gap and tested effects of mate density on male mating decisions in the orb-web spider Argiope bruennichi. Males of this species mutilate their genitalia during copulation, which reduces sperm competition and limits their mating rate to a maximum of two females (bigyny). The mating rate is frequently further reduced by female aggression and cannibalization. Males can reduce the risk of cannibalism if they jump off the female in time, but will then transfer fewer sperm. An alternative solution of this trade-off is to copulate longer, commit self-sacrifice and secure higher minimal paternity. The selfsacrificial strategy may be adaptive if prospective mating chances are uncertain. In $A$. bruennichi, this uncertainty may arise from quick changes in population dynamics. Therefore, we expected that males would immediately respond to information about low or high mate availability and opt for self-sacrifice after a single copulation under low mate availability. If male survival depends on information about prospective mating chances, we further predicted that under high mate availability, we would find a higher rate of males that leave the first mating partner to follow a bigynous mating strategy.

Method. We used naïve males and compared their mating decisions among two treatments that differed in the number of signalling females. In the high mate availability treatment, males perceived pheromone signals from four adult, virgin females, while in the low mate availability treatment only one of four females was adult and virgin and the other three were penultimate and unreceptive.

Results. Males took more time to start mate searching if mate availability was low. However, a selfsacrificial strategy was not more likely under low mate availability. We found no effects of treatment on the duration of copulation, the probability to survive the first copulation or the probability of bigyny. Interestingly, survival chances depended on male size and were higher in small males.

Discussion. Our results do not support the hypothesis that mate density variation affects male mating investment, although they clearly perceived mate density, which they presumably assessed by pheromone quantity. One reason for the absence of male adjustments to mating tactics could be that adaptations to survive female attacks veil adaptations that facilitate mating decisions. 
1 Mate availability does not influence mating strategies in males of the sexually cannibalistic

2 spider Argiope bruennichi

3 Anna-Lena Cory $^{1}$, Jutta M. Schneider ${ }^{1}$

$4 \quad{ }^{1}$ Institute of Zoology, Universität Hamburg, Hamburg, Germany

5

6 Corresponding author:

7 Anna-Lena Cory ${ }^{1}$

8 Email address: anna_lena.cory@yahoo.de

9 


\section{Abstract}

Background. Sexual selection theory predicts that male investment in a current female should be a function of female density and male competition. While many studies have focused on male competition, the impact of female density on male mating investment has been widely neglected. Here, we aimed to close this gap and tested effects of mate density on male mating decisions in the orb-web spider Argiope bruennichi. Males of this species mutilate their genitalia during copulation, which reduces sperm competition and limits their mating rate to a maximum of two females (bigyny). The mating rate is frequently further reduced by female aggression and cannibalization. Males can reduce the risk of cannibalism if they jump off the female in time, but will then transfer fewer sperm. An alternative solution of this trade-off is to copulate longer, commit self-sacrifice and secure higher minimal paternity. The self-sacrificial strategy may be adaptive if prospective mating chances are uncertain. In A. bruennichi, this uncertainty may arise from quick changes in population dynamics. Therefore, we expected that males would immediately respond to information about low or high mate availability and opt for self-sacrifice after a single copulation under low mate availability. If male survival depends on information about prospective mating chances, we further predicted that under high mate availability, we would find a higher rate of males that leave the first mating partner to follow a bigynous mating strategy.

Method. We used naïve males and compared their mating decisions among two treatments that differed in the number of signalling females. In the high mate availability treatment, males perceived pheromone signals from four adult, virgin females, while in the low mate availability treatment only one of four females was adult and virgin and the other three were penultimate and unreceptive.

Results. Males took more time to start mate searching if mate availability was low. However, a self-sacrificial strategy was not more likely under low mate availability. We found no effects of treatment on the duration of copulation, the probability to survive the first copulation or the probability of bigyny. Interestingly, survival chances depended on male size and were higher in small males. 
40 Discussion. Our results do not support the hypothesis that mate density variation affects male mating investment, although they clearly perceived mate density, which they presumably assessed by pheromone quantity. One reason for the absence of male adjustments to mating tactics could be that adaptations to survive female attacks veil adaptations that facilitate mating decisions.

\section{Introduction}

Mating decisions of males often reflect the general trade-off between increasing either mating rates or the investment in the current mate (Magrath \& Komdeur 2003; Scharf et al. 2013; Trivers 1972). The trade-off results from the risk of losing paternity to rival males, since females are often choosy and polyandrous (Arnqvist \& Nilsson 2000; Jennions \& Petrie 1997; Jennions $\&$ Petrie 2000; Parker 1970). Strategies that secure or optimize paternity gains by increasing their reproductive effort are mate guarding, nuptial feeding, mate plugging, courtship behaviour, male fighting, and ejaculate expenditure (Alatalo et al. 1991; Andersson 1994; Eberhard 1994; Uhl et al. 2010; Vahed 1998; Wedell et al. 2002). However, increased reproductive effort has costs such as the depletion of resources (e. g. the amount of sperm), or the consumption of time and energy (Cordts \& Partridge 1996; Hughes et al. 2000; Saeki et al. 2005; Sparkes et al. 1996). Accordingly, males that allocate more time, energy or resources to one mating partner, risk depleting reserves for future mates and missing further mating opportunities. Hence, the decision whether males should invest in the current or future mating partners should strongly depend on mating opportunities (Kokko \& Rankin 2006; Kvarnemo \& Ahnesjo 1996).

Mating opportunities for males are a function of female density and male competition (Kvarnemo \& Ahnesjo 1996), although many studies focus on the latter. There is ample evidence that males invest more in mate guarding, nuptial gifts, and/or ejaculates in response to a strong risk of male competition (Bailey et al. 2010; Carroll \& Corneli 1995; DelBarco-Trillo 2011; Weir et al. 2011). In contrast, the effects of female density on male mating investment are rarely investigated, and if considered they are often veiled in studies regarding population densities or sex ratios (Barrett et al. 2014; Gage 1995; Kokko \& Rankin 2006; Lauer et al. 1996; Linklater et al. 2007; Oku 2009). Studies about sex ratios indeed depict the actual mating opportunities but disregard that under natural conditions, gathering information about mating opportunities may be 
constrained by the male's inability to perceive social cues. For instance, in mating systems where males are adapted to locate females by female sex pheromones or sex-specific chemical cues (Wyatt 2014), males may not necessarily perceive sex-specific chemical cues of conspecific males. In this scenario, males are not able to determine the current sex ratio but can assess female density with some accuracy.

Female localization through chemical cues (or sex pheromones) is particularly common in moths, mantises, and spiders (Gaskett 2007; Greenfield 1981; Holwell et al. 2007; Maxwell et al. 2010; Schulz 2013). Some members of these taxa are known to assess female traits such as body condition or mating status by using chemical cues (e. g. Barry 2010; Foster \& Johnson 2011; Stoltz et al. 2007). Furthermore, in several spider species males developed faster if they received volatile or silk-based pheromonal cues produced by females (Cory \& Schneider 2018; Kasumovic \& Andrade 2006; Neumann \& Schneider 2016). These findings imply that chemical cues are also involved in the assessment of female availability. Other studies suggest that males adjust their mating investment in response to experience with chemical cues produced by females. For example, it was found that-males of the cellar spider Pholcus phalangioides courted longer if they had experienced silk cues of non-virgin, but receptive females (Hoefler 2007). However, it is unknown whether males also adjust their mating investment in the current mate immediately on the receipt of chemical information about female availability. Immediate responses to social information may be particularly relevant in species where the availability of mating partners can be very dynamic throughout the mating season, as is the case in some wellstudied orb-web spiders (Foellmer \& Fairbairn 2005; Zimmer et al. 2012).

In many spiders, males show an extremely high limitation in mating rates because, with each mating encounter, males risk being cannibalized by the female (Elgar \& Crespi 1992). For instance, in the orb-web spider Argiope bruennichi, 50-80\% of males die after a single copulation (Fromhage et al. 2003; Welke et al. 2012). The causes of sexual cannibalism are still debated and are diverse (Elgar \& Schneider 2004; Schneider 2014; Wilder et al. 2009). Some studies have found that sexual cannibalism depends on the hunger state of females and may be an adaptation to food limitation in nature (Berning et al. 2012; Moskalik \& Uetz 2011). Apart from ecological reasons, sexual cannibalism may also be considered a manifestation of sexual conflict (Schneider 2014). While some studies suggest that sexual cannibalism give females control over 
100

101

102

103

104

105

106

107

108

109

110

111

112

113

114

115

116

117

118

119

120

121

122

123

124

125

126

127

128

129

mating (Anderson \& Hebets 2017; Schneider et al. 2006), other studies indicate that males actively sacrifice their life (Andrade 1996; Schneider et al. 2006; Schwartz et al. 2013). A male benefits from self-sacrifice if his body contributes to the female's fecundity or offspring survival and if paternity security is high (Elgar \& Nash 1988; Welke \& Schneider 2012). However, as with any reproductive effort, self-sacrifice has to be traded off against future mating opportunities and should, therefore, depend on female availability (Fromhage \& Jennions 2016).

Extreme male mating investment including self-sacrifice has evolved independently in some spider taxa limiting males to maximally two mating chances (Schneider \& Fromhage 2010). The limitation arises because their paired genitalia (pedipalps) also function as mating-plugs in the equally paired female genital openings (Uhl et al. 2010). Mate plugging by genital mutilation may indeed prevent insemination by rivals but limits males to a single use of each pedipalp (Uhl et al. 2010). Accordingly, males can only invest in one (monogyny) or two females (bigyny). A mating system, in which bigyny and male sacrifice behaviour coexist, provides a good opportunity to test effects of female availability because poor mating decisions have severe fitness consequences.

To test predictions about the density-dependent reproductive investment of males, we used the highly cannibalistic $A$. bruennichi, in which males show monogynous and bigynous mating tactics (Fromhage et al. 2003; Welke et al. 2012). Females stereotypically attack males during copulation, but males can reduce the risk of cannibalization if they copulate briefly and try to jump off in time (Fromhage et al. 2003; Schneider et al. 2005; Welke \& Schneider 2010). By decreasing copulation duration, males also reduce the amount of sperm they transfer (Schneider et al. 2006). The trade-off between sperm transfer and mortality risk is further complicated because a considerable risk of mortality remains even after a short copulation (Fromhage et al. 2003; Welke \& Schneider 2010). Accordingly, self-sacrifice after a long copulation (more than 10 seconds) can be considered a comparably low-risk strategy because the outcome is predictable and $50 \%$ of the life-time sperm load can be transferred (Fromhage et al. 2003; Welke \& Schneider 2010). On the other hand, surviving males have the chance to either minimize the risk of sperm competition in the first female by plugging the second genital opening of the same female or to continue mate searching (bigyny) (Nessler et al. 2007; Welke et al. 2012). A successful bigynous strategy can enhance the overall fitness of a male, but a single copulation 
130 leaves one female genital opening unsecured (Nessler et al. 2007; Welke et al. 2012). Moreover,

131 bigynous males also need to find another mating partner, which should strongly depend on

132 female availability (Fromhage \& Schneider 2012). Thus, under a low density of females, it may

133 be better to increase the investment in the current mating partner particularly if male competition

134 is high. Strong male competition is generally expected in such mating systems because they are

135 associated with extreme female-biased sexual size dimorphism (females more than twice as large

136 as males; Hormiga et al. 2000; Scharff \& Coddington 1997) and reduced mortality of males

137 during development, leading to a male-biased sex ratio (Danielson-Francois et al. 2012; Foellmer

138 \& Fairbairn 2005; Fromhage et al. 2005; Fromhage \& Schneider 2005).

139 For our experiments, we used males that had no experience with female cues until we tested their

140 mating decisions in response to different levels of female density. To manipulate information

141 about female density, we made use of the fact that in A. bruennichi only adult, virgin females

142 produce a sex pheromone that attracts males (Chinta et al. 2010). We conducted mating tests in

143 which males were exposed to either four adult, virgin females (high mate availability) or one

144 adult, virgin and three penultimate females (low mate availability) that do not produce the sex

145 pheromone. We chose the term "mate availability" instead of "female density" because we used

146 the same total female density for both treatments. We predicted that under low mate availability,

147 copulation duration and the likelihood of sexual cannibalism would be higher than under high

148 mate availability. Since our predictions are based on the assumption that males adjust their

149 mating investment to the chance of finding prospective mates, we also predicted that if mate

150 availability and thus prospective mating chances are low, males would rather stay with the

151 current mate than continue mate searching.

152

\section{Material and Methods:}

Spider husbandry:

We used laboratory-bred Argiope bruennichi that derived from 37 egg sacs and whose parents

157 originated from Southern France (Cory \& Schneider 2018). All spiders were kept in cups that

158 were turned upside down and had a hole filled with cotton wool. Also, the cups were roughened 
159 from the inside to facilitate web-building inside the cups. Cup volume (from $50 \mathrm{ml}$ to 11 ) and

160 food provision was adjusted to the spiders' body size. Small spiders received pollen and

161 approximately three dead Drosophila melanogaster three times a week. Two days a week, larger

162 individuals received ca. 20 Drosophila spp. and large subadult and adult females were fed with

163 three Calliphora sp. Spiders were provisioned with water six times a week. Generally, we

164 sprayed water inside the cups; but on days after food provision, we only watered the cotton wool

165 to prevent the prey from escaping. During water provision, we also checked for moults of

166 penultimate males and females and registered the day of maturation. Penultimate and adult

167 spiders can be recognized and distinguished by their genitalia. Males develop a genital bulb on

168 the distal part of each of their pedipalps (Uhl et al. 2007). The genital bulb is undifferentiated in

169 penultimate males whereas it shows discrete sclerotized structures in adult males. Females can be

170 identified by the scape, which is a sclerotized structure covering the epigyne (Uhl et al. 2007).

171 The scape already starts to develop during the penultimate stage but is shorter and less

172 differentiated than in adult females. Furthermore, the scape of penultimate females is covered by

173 a thin membrane and is only exposed after females moult to sexual maturity. Females of both

174 maturation states were put in frames (trapezoidal shape: upper width $14.5 \mathrm{~cm}$, lower width $9 \mathrm{~cm}$,

175 length $13 \mathrm{~cm}$ ) that could be fixed in our test arenas (see experimental design), which offered

176 males access to the females during the mating tests.

177 Spiders either died a natural death in the laboratory and/or were frozen by $-80^{\circ} \mathrm{C}$. Some males 178 were sexually cannibalized during the mating tests, which conforms to the natural situation. We 179 used the tibia-patella length as an index for body size. To measure leg length, we photographed 180 the legs under a binocular and used the measuring tools in the software Leica Application Suite 181 V4.6 (Leica Microsystems ((Switzerland) Limited). In addition to body size, which is fixed after 182 maturation, we also determined the weight shortly after maturation and on the test day with a 183 calibrated scale (Mettler Toledo AB54-S; accuracy $0.1 \mathrm{mg}$ ).

Treatment groups:

186

We tested mating decisions of naïve males with no prior experience to female cues in two

187 experimental set-ups with either a low or high availability of mating partners. In both treatments, 
188 males were exposed to four females, but we manipulated the number of pheromone-emitting 189 females.

190 The low mate availability treatment was composed of one adult, virgin and three penultimate 191 females. We know from previous studies in A. bruennichi that adult, virgin females are highly 192 receptive whereas penultimate females are not (Schneider et al. 2006, personal obs).

193 Furthermore, subadult females do not produce the sex pheromone that was found on the body 194 and silk of adult, virgin females and that attracted males (Chinta et al. 2010).

195 In the high mate availability treatment all females were adult and virgin. The four females in 196 each high mate availability treatment were matched for adult age (we only allowed an age 197 difference of two days) because previous studies found that mate attraction depends on female 198 age (Cory \& Schneider 2016). We ensured that the mean ages of the four females in the high mate availability treatment did not differ from the ages of the adult, virgin females in the low mate availability treatment (Mann Whitney $\mathrm{U}$ test: $\mathrm{N}=50, \mathrm{~W}=288, \mathrm{p}=0.8167$ ).

While we controlled for the adult age, we were unable to match for body size, which is a common measure of female quality. To minimize potential effects of female quality, we used individuals from Southern France for which previous studies found that female body size had no effect on male mating decisions (Cory \& Schneider 2018). Males generally mated with the first female they encountered. Body sizes of the females that copulated in both treatments revealed no difference ( $\mathrm{t}$ test: $\mathrm{N}=50, \mathrm{df}=48, \mathrm{t}=-0.7504, \mathrm{p}=0.4567)$.

Completion of the treatments required a considerable number of penultimate and virgin adults of similar age so that we had to use some females multiple times. We ensured that we only re-used females that had no contact with males in previous tests. Males and females were always unrelated, but in some tests, two of the four females were siblings.

Test arenas:

213 The tests were conducted in test arenas (Fig. 1) simulating semi-natural conditions in which 214 females were randomly distributed and males could move freely. The floor of the arenas was 215 covered with fresh packing paper, on which males could easily walk. The backend of the arena 
216 had an air permeable window through which a ventilator produced a slight airflow to ensure

217 pheromone flow within the arena. The starting point for males was at the front end in a central

218 position and consisted of a roughened plastic stick positioned with an inclination of

219 approximately $45^{\circ}$. Each test arena was fitted with the same four applications to fix the frames

220 with the females in identical positions (front, back and two in the middle). In the high mate

221 availability treatment, the four females were randomly positioned on the four spots. In the low

222 mate availability tests, the single adult, virgin female was always located in the front position

223 closest to the male starting point, and the three penultimate females were randomly distributed to 224 the remaining positions.

226 Test procedure:

227 Between July 14 and August 16 2016, the experiments took place outside on the roof of the Institute of Zoology of the Universität Hamburg. We monitored the mating tests in up to eight test arenas that were placed underneath a waterproof pavilion roof. In clean and prepared arenas, males were gently placed on the top of the roughened plastic stick (starting point). The time males left the plastic stick was defined as the start of mate searching. Males that did not start their mate search within 45 minutes were replaced. Males that were caught at the edges of the arena were reintroduced to the starting point and the clock was restarted. Males that ended up in the web of a penultimate female in the low mate availability treatment were given the chance to leave this female for 45 minutes until they were reintroduced to the starting point. However, males that visited penultimate females might also choose an opportunistic mating strategy, in which they mate with females while they moult to sexual maturity and are defenceless (Uhl et al. 2015). To take this into account, we analysed our data with and without males that had contact to penultimate females. Since the results did not deviate from each other, we only present the analysis with the total sample size and provide the other results as supplemental material (S1).

During the mating tests, we monitored which female was visited, the duration of copulation and whether the male was cannibalized or not. We also scored whether surviving males copulated a second time with the first mating partner (monopolization) or whether they left the female. We defined that males left the female when there was no contact with the web or frame of the female 
245

and a clear absence of male dragline silk attachments for at least one hour. In such cases, we presumed an intention to continue mate searching and follow a bigynous tactic.

\section{Statistical analysis:}

We used R (R Developmental Core Team 2014) for data analyses. We performed parametric tests for normally distributed data and otherwise non-parametric tests.

\section{Mate searching and localization:}

Our predictions rely on the assumption that males estimate the density of adult virgin females via sex pheromone quantity. Thus, mate searching and localization should also be influenced by the number of signalling females. We tested whether latencies until mate search started and the probability of reintroduction after 45 minutes differed between treatments. Several previous studies from our lab found no evidence for sequential male mate choice in A. bruennichi (Schulte et al. 2010). We nevertheless explored with binomial tests whether male mate search was directed towards the largest, most attractive female, or to the closest female.

\section{Copulation duration and sexual cannibalism:}

We generated multivariate models to test influences of main effects and covariates on different dependent variables. We simplified the model by removing one variable at a time. Afterwards, we compared each simplified model with the original model using likelihood ratio tests. One by one, we omitted all variables that led to non-significant changes of deviance and removed the least-significant variable first. In the end, we kept the model that successfully explained more variation in the data than the other models.

To test whether copulation duration depended on mate availability, we applied a Cox regression model using the package "survival" (Therneau 2014). As further explanatory variables, we included female size (tibia-patella length), female condition (residuals of the linear regression between female test weight and female size; see Jakob et al. 1996) and the relative weight 
272 change of males between adulthood and test day. We used the relative weight change rather than

273 the residual-based male condition that we used for females because earlier studies found a

274 significant effect on male mating decisions (Cory \& Schneider 2018). In a separate Cox

275 regression model with a reduced sample size (we could not measure the legs of four males), we

276 also tested whether male size affected copulation duration.

277 To test whether mate availability affected the probability of sexual cannibalism, we applied a

278 binary logistic regression. We used the same female and male traits as above.

279

Mating decisions in surviving males:

281 The sample size of surviving males was fairly small, which was why we only conducted a Fisher exact test to explore whether males of the low mate availability treatment would rather stay with the first mating partner than to continue mate searching.

\section{Results:}

Mate searching and localization:

287

288

289

290

291

292

293

294

295

296

297

298

In the low mate availability treatment, males started mate searching significantly later than in the high mate availability treatment (high mate availability: median=32 s, interquartile range=184 s; low mate availability: median: $266 \mathrm{~s}$, interquartile range $=580 \mathrm{~s}$; Mann-Whitney $\mathrm{U}$ test: $\mathrm{N}=50$, $\mathrm{W}=202, \mathrm{p}=0.0328$ ). Moreover, we found a tendency that males were more disoriented in the low mate-availability treatments, so that males had to be reintroduced to the starting point after 45 minutes more frequently than males in the high mate availability treatment (high mate availability: $16 \%$; low mate availability: $56 \%$; Pearson $\mathrm{Chi}^{2}$ test: $\mathrm{N}=50, \mathrm{Chi}^{2}=3.4286$, $\mathrm{p}=0.0641)$.

In the low mate availability treatment, 7 of 25 males visited a penultimate female first. A prior visit of a penultimate female had no effect on the rate of cannibalism (Fisher exact test: $\mathrm{N}=25$, $\mathrm{p}=0.6592$ ) or on the duration of copulation (Mann-Whitney $U$ test: $N=25, W=76, p=0.4584$ ) with the receptive female. In the high mate availability treatment, $48 \%$ of males mated with the 
299 closest female in the arena, which deviates significantly from a $25 \%$ chance (Binomal test:

$300 \mathrm{~N}=25, \mathrm{p}=0.0178$ ). We found no evidence that males were more likely to visit the heaviest female

301 (Binomal test: $\mathrm{N}=25, \mathrm{p}=0.4872$ ).

302

303 Copulation duration and cannibalism rate:

304 Most males (90\%) copulated less than 15 seconds (Fig 2A) and one outlier male from the high

305

306

307

308

309

310

311

312

313

314

315

316

317

318

319

320

321

322

323

324

325 mate availability treatment copulated for 113 seconds. The duration of copulation was not affected by mate availability or any other effect that we tested (Table 1) regardless of whether the outlier was included or not. Therefore, we kept this male in the final analysis. Unlike in previous studies using different populations from the same species (Fromhage et al. 2003; Welke \& Schneider 2010), longer copulation duration was not significantly associated with the probability of sexual cannibalism (Mann-Whitney $\mathrm{U}$ test: $\mathrm{N}=49, \mathrm{~W}=327, \mathrm{p}=0.3944$ ). The overall rate of cannibalism was ca. $60 \%$ and did not significantly vary with mate availability (Fig 2B; Table 2). The only significant predictor of sexual cannibalism was a large body size of males (Table 2, Fig. 3).

Mating decisions in surviving males:

$63.2 \%$ of the surviving males remated and monopolized the first mating partner. While nearly all males ( 8 of 10) stayed with the first mating partner in the low mate availability treatment, only four of the nine surviving males monopolized the female under high mate availability. However, the likelihood of monopolization did not significantly differ between treatments (Fig 2C; Fisher exact test: $\mathrm{N}=19, \mathrm{p}=0.1698$ ), which might be due to the very small sample size.

\section{Discussion:}

Theory predicts that mating opportunities should strongly influence mating investment (Kokko \& Rankin 2006; Trivers 1972). In Argiope bruennichi, males can increase their mating investment by prolonging copulation duration, which also increases the risk of sexual 
326 cannibalism. Due to their short mating season (Zimmer et al. 2012), we expected that males

327 would quickly respond to current information about mate availability. We predicted that under a

328 low availability of adult, virgin females, males would copulate longer and be more often

329 cannibalized than under a high availability of adult, virgin females. Under the latter condition,

330 survival and a bigynous mating tactic would yield the largest fitness return. However, we found

331 no evidence for a strategic adjustment of mating tactic to different levels of mate availability

332 during mate searching.

333 Contrary to our predictions, neither the probability of sexual cannibalism nor the duration of

334 copulation differed between high or low mate availability. Also, there was no significant

335 evidence that males would leave the first mating partner after the first copulation and presumably

336 engage a bigynous mating tactic when additional adult virgins were nearby. This was unexpected

337 because low mate availability renders a bigynous mating tactic risky and a self-sacrificial

338 strategy with a single, long copulation should be the safer option to enhance male fitness. Even

339 though frequency differences complied with the expected direction, sample size and power were

340 likely too small to statistically verify the effect.

341 Despite our failure to detect any effect of mate availability on male mating investment, we

342 suggest that variation in female availability is biologically relevant for A. bruennichi males.

343 Argiope bruennichi is widespread in Europe and can occupy meadows in high densities

344 (Bruggisser et al. 2012; Krehenwinkel \& Tautz 2013; Kumschick et al. 2011). While the spatial

345 distribution of females may not be a problem, males have to deal with temporal changes in the

346 availability of adult, virgin females (Zimmer et al. 2012). As in many spider species, the

347 availability of adult, virgin females is very low, especially in the beginning of the mating season,

348 and the protandrous males strongly compete for access to the early maturing females (Elias et al.

349 2011). Moreover, the combination of male scramble competition and low mate rejection in

350 females (Schneider et al. 2006) leads to a swift disappearance of virgin females from the mating

351 pool. Thus, the density of adult, virgin females is very dynamic throughout the season, and it

352 should be adaptive for males to integrate social information in their mating decisions. This might

353 be most important for populations in Northern Europe where the mating season is very short.

354 However, in this study, we used spiders derived from Mediterranean populations where seasonal

355 constraints and competition may be less severe. We chose individuals from Southern Europe 
356 because unlike in Northern males, their mating decisions are not influenced by female body size

357 (Cory \& Schneider 2018), for which we could not control in our high mate availability treatment.

358 In addition to this difference, Southern males also respond to female cues and shorten their

359 developmental time to the presence of those (Cory \& Schneider 2018).

360 In contrast to our experiment, most studies tested effects of social experience on mating

361 decisions. Here, individuals received social information (e. g. absence and presence of social

362 cues) for a much longer period of time than in our study. It is well established that social

363 information during development can affect life history traits and mating decisions (Kasumovic \&

364 Brooks 2011; Rodriguez et al. 2013) such as mating preferences (Hebets 2003; Rutledge et al.

365 2010; Stoffer \& Uetz 2016). For instance, it was found that females of the wolf spider

366 Schizocosa ocreata were more choosy as adults if they had a high encounter rate of males during

367 development (Stoffer \& Uetz 2015). Hence, mate availability might have a strong impact on

368 mating decisions, but individuals may need enough time to process social information.

369 We argue that male mating investment will strongly rely on information about adult, virgin

370 female availability, particularly in mating systems where males are adapted to locate receptive

371 females by sex pheromones. To account for this, we manipulated the number of adult, virgin

372 females while leaving the density of webs with females constant. We used penultimate females

373 in the low mate availability treatment because they do not produce the pheromone known for

374 adult, virgin females (Chinta et al. 2010). However, absence and presence of a pheromone and

375 the ability to distinguish do not necessarily mean that males cannot perceive the presence of

376 penultimate females. In the field, males are often found in the vicinity of penultimate females

377 and are known to copulate with them during their final moult (Zimmer \& Schneider 2016). This

378 is beneficial for males because there is no risk of sexual cannibalism from a freshly moulted

379 mate (Uhl et al. 2015). Accordingly, penultimate females do indeed promise future mating

380 opportunities.

381 Although many studies found that spider males encounter penultimate females (Bel-Venner \&

382 Venner 2006; Biaggio et al. 2016; Erez et al. 2005; Johnson 2005), there is ample evidence that

383 males more easily detect adult virgins than immature or mated females (Gaskett 2007). In many

384 species, including $A$. bruennichi, males recognize adult, virgin females by sex pheromones that

385 are absent in subadult and mated females (Chinta et al. 2010; Gaskett 2007; Thomas 2011). In 
386 line with this, behavioural choice tests with $A$. bruennichi confirmed a preference for virgin 387 females (Schneider et al. 2016; Schulte et al. 2010). Similar results were found in other spider

388

389

390

391

392

393

394

395

396

397

398

399

400

401

402

403

404

405

406

407

408

409

410

411

412

413

414

415 species (Stoltz et al. 2007; Tuni \& Berger-Tal 2012; Watson 1986). For instance, males of Stegodyphus lineatus showed less searching behaviour when presented to immature females than to adult, virgin females (Tuni \& Berger-Tal 2012). Our results agree with this finding. Under the low mate availability treatment, males waited longer until they moved and needed more attempts to find a female compared to males under high mate availability. This suggests that male sensory organs respond to increased quantity of pheromones.

Our low mate availability treatment simulated the beginning of the mating season, in which males could expect future mating chances with adult, virgin females. As the mating season proceeds, future mating chances become increasingly scarce because most females have moulted to adulthood and mated (Zimmer et al. 2012). Thus, we suggest that future studies should introduce a control treatment with double-mated females instead of penultimate females to simulate conditions late in the season. While females that had copulated once still offer mating opportunities, double-mated females have both copulatory openings plugged and in $A$. bruennichi, copulations into plugged genital openings are unlikely to return paternity gains (Nessler et al. 2007). We further suggest a second control-group, which only consists of a single adult, virgin female to test whether males are generally able to assess the presence of females independent of their developmental and mating state.

Another reason for our negative results could be that information about mate availability is not a reliable predictor of fitness, particularly in the absence of information about the degree of male competition. While it seems likely that $A$. bruennichi males can estimate mate availability by female sex pheromones, it is unknown whether males receive cues from other males to make informed decisions. Simultaneous mate choice experiments showed that $A$. bruennichi males do not modify their mate choice decisions in the presence of silk cues from rival males (Schneider et al. 2016). On the other hand, it was found that males decrease the duration of courtship if a rival male is present in the female web. Similarly, males copulated longer and more likely plugged the female in the presence of a rival in the closely related species Argiope lobata (Hirt et al. 2017). We, therefore, propose that future studies with A. bruennichi should integrate information on male density either by testing it as a single factor or in relation to the sex ratio. 
416 Contrary to several previous studies on A. bruennichi, we could not confirm the well-established

417 positive relationship between copulation duration and cannibalism risk (Fromhage et al. 2003;

418 Nessler et al. 2009; Schneider et al. 2006; Schneider \& Lesmono 2009; Welke \& Schneider

419 2010). We suggest that this interesting difference may be explained by differences in body size

420 between populations. All previous studies used Northern populations, while we used individuals

421 from the Mediterranean area that are larger and more heterogeneous in body size (Cory \&

422 Schneider 2018; Krehenwinkel \& Tautz 2013). Since size differences between sexes matter in

423 sexually cannibalistic spiders (Wilder \& Rypstra 2008), such differences might have affected our

424 results. In contrast to previous studies, we detected size dependent male survival and a benefit of

425 small male size. Observations suggest that larger males were less likely to escape because

426 females were more successful in grasping larger males. A small size advantage of males in

427 avoiding sexual cannibalism was already shown in other studies with spiders (Elgar et al. 2000;

428 Schneider \& Elgar 2001), although the opposite was also found (Elgar \& Jones 2008; Elgar et al.

429 2000; Persons \& Uetz 2005). Possibly, size related survival differences between males have

430 obscured the relationship between copulation duration and sexual cannibalism.

431 To our knowledge, this study is the first that tested immediate effects of different levels of mate

432 availability on male investment in a mating system that strongly relies on chemical

433 communication. Although chemical communication is involved in mating interactions of many

434 species, the use of chemical cues is poorly understood (Wyatt 2014). In comparison, we find

435 many studies on mating systems where males can assess female availability by vision (Barrett et

436 al. 2014; Berglund 1995; Lauer et al. 1996). For instance, it was found that pipefish males

437 (Syngnathus typhle) were only choosy if they were exposed to a high density of females during

438 decision-making (Berglund 1995). In line with this, a study on social experience in guppies

439 (Poecilia reticulata) exposed males visually to a low or high female availability and found that,

440 thereafter, large males showed a higher sexual interest in the current female if other mating

441 opportunities were absent (Barrett et al. 2014). In contrast, water strider males (Aquarius

442 remiges), that were housed under different density treatments with either females or males, only

443 adjusted their pre-copulatory mating effort if they had experienced a high density of males before

444 (Lauer et al. 1996). Independent of whether social information was manipulated during or before

445 male mating decisions were tested, this diversity of findings shows that future research should be 
446 based on an integrated framework of information about mate perception, mating constraints and 447 ecological conditions.

448

449

450

451

452

453

454

455

456

457

458

459

460

461

462

463

464

465

466

467

468

469

470

471

472

\section{Conclusion}

Life history theory predicts a trade-off between the current and the future mating effort (Stearns 2000). Which balance of investment maximizes fitness of males should strongly depend on prospective mating opportunities (Kokko \& Rankin 2006). However, adaptations to adjust investment to mate availability may only evolve if males can gain sufficient information about their social environment. Little is known about information use in a sensory world based on volatile chemicals particularly in the context of mating strategies. Because of their low maximal mating rate, males of sexually cannibalistic spiders are under strong selection to optimise their investment. More studies on such systems may proof useful to further our understanding of chemical information and how it is integrated into decision making. While several studies found that males gain social information through chemical cues by females (Gaskett 2007; Greenfield 1981; Holwell et al. 2007; Maxwell et al. 2010; Schulz 2013), it is much less clear whether males can detect chemical cues of rival males. More studies are desired that address whether and how males can perceive rivals and integrate competition in their mating decision. For instance, male spiders may assess other males either by chemical cues or by silk threads that males leave behind during mate search. Besides proximate reasons, ecological and behavioural constraints resulting from the mating system (e. g. mating systems with sexual cannibalism) may also influence adaptations and prevent that males will flexibly respond to mate availability. Here, empirical studies could test whether an interaction between mate availability and risk of sexual cannibalism influences male mating decisions.

\section{Acknowledgements}

We thank Julia Becker, Tomma Dirks, Sebastian Franke, Onno Preik, Angelika Taebel-Hellwig and Leonie Schaum for their help with animal husbandry. We are particularly grateful to Tomma 
473 Dirks and Leonie Schaum for their support during the experimental phase. We thank Stefanie

474 Zimmer, Brent Stoffer and two anonymous referees for valuable comments on our manuscript.

475

476

\section{References:}

477

478

479

480

481

482

483

484

485

486

487

488

489

490

491

492

493

494

495

496

497

498

499

500

501

502

503

504

Alatalo RV, Höglund J, and Lundberg A. 1991. Lekking in the black grouse - a test of male viability Nature 352:155-156. 10.1038/352155a0

Anderson AG, and Hebets EA. 2017. Increased insertion number leads to increased sperm transfer and fertilization success in a nursery web spider. Animal Behaviour 132:121-127. 10.1016/j.anbehav.2017.08.007

Andersson M. 1994. Sexual Selection. Princeton, New Jersey: Princeton University Press.

Andrade MCB. 1996. Sexual selection for male sacrifice in the Australian redback spider. Science 271:70-72. 10.1126/science.271.5245.70

Arnqvist G, and Nilsson T. 2000. The evolution of polyandry: multiple mating and female fitness in insects. Animal Behaviour 60:145-164. 10.1006/anbe.2000.1446

Bailey NW, Gray B, and Zuk M. 2010. Acoustic experience shapes alternative mating tactics and reproductive investment in male field crickets. Current Biology 20:845-849. 10.1016/j.cub.2010.02.063

Barrett LT, Evans JP, and Gasparini C. 2014. The effects of perceived mating opportunities on patterns of reproductive investment by male guppies. Plos One 9. 10.1371/journal.pone.0093780

Barry KL. 2010. Influence of female nutritional status on mating dynamics in a sexually cannibalistic praying mantid. Animal Behaviour 80:405-411. 10.1016/j.anbehav.2010.05.024

Bel-Venner MC, and Venner S. 2006. Mate-guarding strategies and male competitive ability in an orbweaving spider: results from a field study. Animal Behaviour 71:1315-1322. 10.1016/j.anbehav.2005.08.010

Berglund A. 1995. Many mates make male pipefish choosy. Behaviour 132:213-218. $10.1163 / 156853995 \times 00702$

Berning AW, Gadd RDH, Sweeney K, MacDonald L, Eng RYY, Hess ZL, and Pruitt JN. 2012. Sexual cannibalism is associated with female behavioural type, hunger state and increased hatching success. Animal Behaviour 84:715-721. 10.1016/j.anbehav.2012.06.030

Biaggio MD, Sandomirsky I, Lubin Y, Harari AR, and Andrade MCB. 2016. Copulation with immature females increases male fitness in cannibalistic widow spiders. Biology Letters 12.

$10.1098 / \mathrm{rsbl} .2016 .0516$ 
505 Bruggisser OT, Sandau N, Blandenier G, Fabian Y, Kehrli P, Aebi A, Naisbit RE, and Bersier LF. 2012.

506 Direct and indirect bottom-up and top-down forces shape the abundance of the orb-web spider

507 Argiope bruennichi. Basic and Applied Ecology 13:706-714. 10.1016/j.baae.2012.10.001

508 Carroll SP, and Corneli PS. 1995. Divergence in male mating tactics between 2 populations of the

509

510

511

512

513

514

515

516

517

518

519

520

521

522

523

524

525

526

527

528

529

530

531

532

533

534

535

536

537

538

soapberry bug. 2. Genetic change and the evolution of a plastic reaction norm in a variable socialenvironment. Behavioral Ecology 6:46-56. DOI 10.1093/beheco/6.1.46

Chinta SP, Goller S, Lux J, Funke S, Uhl G, and Schulz S. 2010. The sex pheromone of the wasp spider Argiope bruennichi. Angewandte Chemie-International Edition 49:2033-2036. 10.1002/anie.200906311

Cordts R, and Partridge L. 1996. Courtship reduces longevity of male Drosophila melanogaster. Animal Behaviour 52:269-278. 10.1006/anbe.1996.0172

Cory A-L, and Schneider JM. 2016. Old maids have more appeal: effects of age and pheromone source on mate attraction in an orb-web spider. Peerj 4. 10.7717/peerj.1877

Cory AL, and Schneider JM. 2018. Effects of social information on life history and mating tactics of males in the orb-web spider Argiope bruennichi. Ecology and Evolution 8:344-355. $10.1002 /$ ece 3.3672

Danielson-Francois A, Hou C, Cole N, and Tso IM. 2012. Scramble competition for moulting females as a driving force for extreme male dwarfism in spiders. Animal Behaviour 84:937-945. 10.1016/j.anbehav.2012.07.018

DelBarco-Trillo J. 2011. Adjustment of sperm allocation under high risk of sperm competition across taxa: a meta-analysis. Journal of Evolutionary Biology 24:1706-1714. 10.1111/j.14209101.2011.02293.x

Eberhard WG. 1994. Evidence for widespread courtship during copulation in 131 species of insects and spiders, and implications for cryptic female choice. Evolution 48:711-733. 10.2307/2410481

Elgar MA, and Crespi BJ. 1992. Sexual cannibalism in spiders and other invertebrates. Cannibalism: Ecology and evolution among diverse taxa. p 128-155.

Elgar MA, and Jones TM. 2008. Size-dependent mating strategies and the risk of cannibalism. Biological Journal of the Linnean Society 94:355-363. 10.1111/j.1095-8312.2008.00986.x

Elgar MA, and Nash DR. 1988. Sexual cannibalism in the garden spider Araneus diadematus. Animal Behaviour 36:1511-1517. Doi 10.1016/S0003-3472(88)80221-5

Elgar MA, and Schneider JM. 2004. Evolutionary significance of sexual cannibalism. Advances in the Study of Behavior, Vol 34 34:135-163. Doi 10.1016/S0065-3454(04)34004-0

Elgar MA, Schneider JM, and Herberstein ME. 2000. Female control of paternity in the sexually cannibalistic spider Argiope keyserlingi. Proc Biol Sci 267:2439-2443. 10.1098/rspb.2000.1303 
539 Elias DO, Andrade MCB, and Kasumovic MM. 2011. Dynamic population structure and the evolution of

540

541

542

543

544

545

546

547

548

549

550

551

552

553

554

555

556

557

558

559

560

561

562

563

564

565

566

567

568

569

570

571

572 spider mating systems. In: Casas J, ed. Advances in Insect Physiology, Vol 41: Spider Physiology and Behaviour - Behaviour, 65-114.

Erez T, Schneider JM, and Lubin Y. 2005. Is male cohabitation costly for females of the spider Stegodyphus lineatus (Eresidae)? Ethology 111:693-704. 10.1111/j.1439-0310.2005.01090.x

Foellmer MW, and Fairbairn DJ. 2005. Competing dwarf males: sexual selection in an orb-weaving spider. Journal of Evolutionary Biology 18:629-641. 10.1111/j.1420-9101.2005.00868.x

Foster SP, and Johnson CP. 2011. Signal honesty through differential quantity in the female-produced sex pheromone of the moth Heliothis virescens. Journal of Chemical Ecology 37:717-723.

$$
\text { 10.1007/s10886-011-9982-8 }
$$

Fromhage L, Elgar MA, and Schneider JM. 2005. Faithful without care: The evolution of monogyny. Evolution 59:1400-1405.

Fromhage L, and Jennions MD. 2016. Coevolution of parental investment and sexually selected traits drives sex-role divergence. Nature Communications 7. 10.1038/ncomms12517

Fromhage L, and Schneider JM. 2005. Virgin doves and mated hawks: contest behaviour in a spider. Animal Behaviour 70:1099-1104. 10.1016/j.anbehav.2005.02.020

Fromhage L, and Schneider JM. 2012. A mate to die for? A model of conditional monogyny in cannibalistic spiders. Ecology and Evolution 2:2572-2582. 10.1002/ece3.372

Fromhage L, Uhl G, and Schneider JM. 2003. Fitness consequences of sexual cannibalism in female Argiope bruennichi. Behavioral Ecology and Sociobiology 55:60-64. 10.1007/s00265-003-0656-6

Gage MJG. 1995. Continuous variation in the reproductive strategy as an adaptive response to population density in the moth Plodia interpunctella. Proceedings of the Royal Society B-Biological Sciences 261:25-30. 10.1098/rspb.1995.0112

Gaskett AC. 2007. Spider sex pheromones: emission, reception, structures, and functions. Biological Reviews 82:26-48. 10.1111/j.1469-185X.2006.00002.x

Greenfield MD. 1981. Moth sex pheromones: an evolutionary perspective. Florida Entomologist 64:4-17. Doi $10.2307 / 3494597$

Hebets EA. 2003. Subadult experience influences adult mate choice in an arthropod: Exposed female wolf spiders prefer males of a familiar phenotype. Proceedings of the National Academy of Sciences of the United States of America 100:13390-13395. 10.1073/pnas.2333262100

Hirt K, Ruch J, and Schneider JM. 2017. Strategic male mating behaviour in Argiope lobata. Animal Behaviour 124:27-34. 10.1016/j.anbehav.2016.11.030

Hoefler CD. 2007. Male mate choice and size-assortative pairing in a jumping spider, Phidippus clarus. Animal Behaviour 73:943-954. 10.1016/j.anbehav.2006.10.017 
573 Holwell GI, Barry KL, and Herberstein ME. 2007. Mate location, antennal morphology, and ecology in

574

575

576

577

578

579

580

581

582

583

584

585

586

587

588

589

590

591

592

593

594

595

596

597

598

599

600

601

602

603

604

605

606 two praying mantids (Insecta : Mantodea). Biological Journal of the Linnean Society 91:307-313. 10.1111/j.1095-8312.2007.00788.x

Hormiga G, Scharff N, and Coddington JA. 2000. The phylogenetic basis of sexual size dimorphism in orb-weaving spiders (Araneae, Orbiculariae). Systematic Biology 49:435-462. $10.1080 / 10635159950127330$

Hughes L, Chang BSW, Wagner D, and Pierce NE. 2000. Effects of mating history on ejaculate size, fecundity, longevity, and copulation duration in the ant-tended lycaenid butterfly, Jalmenus evagoras. Behavioral Ecology and Sociobiology 47:119-128. 10.1007/s002650050002

Jakob EM, Marshall SD, and Uetz GW. 1996. Estimating fitness: A comparison of body condition indices. Oikos 77:61-67. 10.2307/3545585

Jennions MD, and Petrie M. 1997. Variation in mate choice and mating preferences: a review of causes and consequences. Biol Rev Camb Philos Soc 72:283-327. 10.1017/s0006323196005014

Jennions MD, and Petrie M. 2000. Why do females mate multiply? A review of the genetic benefits. Biological Reviews 75:21-64. 10.1017/s0006323199005423

Johnson JC. 2005. Cohabitation of juvenile females with mature males promotes sexual cannibalism in fishing spiders. Behavioral Ecology 16:269-273. 10.1093/beheco/arh152

Kasumovic MM, and Andrade MCB. 2006. Male development tracks rapidly shifting sexual versus natural selection pressures. Current Biology 16:R242-R243. 10.1016/j.cub.2006.03.017

Kasumovic MM, and Brooks RC. 2011. It's all who you know: the evolution of socially cued anticipatory plasticity as a mating strategy. Quarterly Review of Biology 86:181-197. Doi 10.1086/661119

Kokko H, and Rankin DJ. 2006. Lonely hearts or sex in the city? Density-dependent effects in mating systems. Philosophical Transactions of the Royal Society B-Biological Sciences 361:319-334. $10.1098 /$ rstb.2005.1784

Krehenwinkel H, and Tautz D. 2013. Northern range expansion of European populations of the wasp spider Argiope bruennichi is associated with global warming-correlated genetic admixture and population-specific temperature adaptations. Mol Ecol 22:2232-2248. 10.1111/mec.12223

Kumschick S, Fronzek S, Entling MH, and Nentwig W. 2011. Rapid spread of the wasp spider Argiope bruennichi across Europe: a consequence of climate change? Climatic Change 109:319-329. 10.1007/s10584-011-0139-0

Kvarnemo C, and Ahnesjo I. 1996. The dynamics of operational sex ratios and competition for mates. Trends in Ecology \& Evolution 11:404-408. 10.1016/0169-5347(96)10056-2

Lauer MJ, Sih A, and Krupa JJ. 1996. Male density, female density and inter-sexual conflict in a streamdwelling insect. Animal Behaviour 52:929-939. 10.1006/anbe.1996.0241 
607 Linklater JR, Wertheim B, Wigby S, and Chapman T. 2007. Ejaculate depletion patterns evolve in 608 response to experimental manipulation of sex ratio in Drosophila melanogaster. Evolution $609 \quad 61: 2027-2034.10 .1111 / j .1558-5646.2007 .00157 . x$

610 Magrath MJL, and Komdeur J. 2003. Is male care compromised by additional mating opportunity?

611 Trends in Ecology \& Evolution 18:424-430. 10.1016/s0169-5347(03)00124-1

612 Maxwell MR, Barry KL, and Johns PN. 2010. Examinations of female pheromone use in two praying 613 mantids, Stagmomantis limbata and Tenodera aridifolia sinensis (Mantodea: Mantidae). Annals 614 of the Entomological Society of America 103:120-127. 10.1603/008.103.0115

615 Moskalik B, and Uetz GW. 2011. Female hunger state affects mate choice of a sexually selected trait in a 616 wolf spider. Animal Behaviour 81:715-722. 10.1016/j.anbehav.2010.12.016

617 Nessler SH, Uhl G, and Schneider JM. 2007. Genital damage in the orb-web spider Argiope bruennichi

618 (Araneae : Araneidae) increases paternity success. Behavioral Ecology 18:174-181.

$619 \quad 10.1093 /$ beheco/ar1074

620 Nessler SH, Uhl G, and Schneider JM. 2009. Scent of a woman - the effect of female presence on sexual

621

622 cannibalism in an orb-weaving spider (Araneae: Araneidae). Ethology 115:633-640.

10.1111/j.1439-0310.2009.01646.x

623 Neumann R, and Schneider JM. 2016. Socially cued developmental plasticity in web-building spiders. 624 Bmc Evolutionary Biology 16. 10.1186/s12862-016-0736-7

625 Oku K. 2009. Effects of density experience on mate guarding behavior by adult male Kanzawa spider 626 mites. Journal of Ethology 27:279-283. 10.1007/s10164-008-0117-9

627 Parker GA. 1970. Sperm competition and its evolutionary consequences in insects. Biol Rev Camb Philos 628 Soc 45:525-\&. 10.1111/j.1469-185X.1970.tb01176.x

629 Persons MH, and Uetz GW. 2005. Sexual cannibalism and mate choice decisions in wolf spiders:

630 influence of male size and secondary sexual characters. Animal Behaviour 69:83-94.

$631 \quad 10.1016 /$ j.anbehav.2003.12.030

632 R Developmental Core Team. 2014. A language and environment for statistical computing. Vienna, 633 Austria: R Foundation for Statistical Computing.

634 Rodriguez RL, Rebar D, and Fowler-Finn KD. 2013. The evolution and evolutionary consequences of 635 social plasticity in mate preferences. Animal Behaviour 85:1041-1047.

$636 \quad$ 10.1016/j.anbehav.2013.01.006

637 Rutledge JM, Miller A, and Uetz GW. 2010. Exposure to multiple sensory cues as a juvenile affects adult 638 female mate preferences in wolf spiders. Animal Behaviour 80:419-426.

$639 \quad 10.1016 /$ j.anbehav.2010.05.027 
640 Saeki Y, Kruse KC, and Switzer PV. 2005. Physiological costs of mate guarding in the Japanese beetle

641

642

643

644

645

646

647

648

649

650

651

652

653

654

655

656

657

658

659

660

661

662

663

664

665

666

667

668

669

670

671

672

673

(Popillia japonica Newman). Ethology 111:863-877. 10.1111/j.1439-0310.2005.01106.x

Scharf I, Peter F, and Martin OY. 2013. Reproductive trade-offs and direct costs for males in arthropods. Evolutionary Biology 40:169-184. 10.1007/s11692-012-9213-4

Scharff N, and Coddington JA. 1997. A phylogenetic analysis of the orb-weaving spider family Araneidae (Arachnida, Araneae). Zoological Journal of the Linnean Society 120:355-434. 10.1111/j.1096-3642.1997.tb01281.x

Schneider JM. 2014. Sexual cannibalism as a manifestation of sexual conflict. Cold Spring Harbor Perspectives in Biology 6. 10.1101/cshperspect.a017731

Schneider JM, and Elgar MA. 2001. Sexual cannibalism and sperm competition in the golden orb-web spider Nephila plumipes (Araneoidea): female and male perspectives. Behavioral Ecology 12:547-552. 10.1093/beheco/12.5.547

Schneider JM, and Fromhage L. 2010. Monogynous mating strategies in spiders. Animal Behaviour: Evolution and Mechanisms:441-464. 10.1007/978-3-642-02624-9_15

Schneider JM, Fromhage L, and Uhl G. 2005. Extremely short copulations do not affect hatching success in Argiope bruennichi (Araneae, Araneidae). Journal of Arachnology 33:663-669. 10.1636/s0332.1

Schneider JM, Gilberg S, Fromhage L, and Uhl G. 2006. Sexual conflict over copulation duration in a cannibalistic spider. Animal Behaviour 71:781-788. 10.1016/j.anbehav.2005.05.012

Schneider JM, and Lesmono K. 2009. Courtship raises male fertilization success through post-mating sexual selection in a spider. Proceedings of the Royal Society B-Biological Sciences 276:31053111. 10.1098/rspb.2009.0694

Schneider JM, Zimmer SM, Gatz AL, and Sauerland K. 2016. Context- and state-dependent male mate choice in a sexually cannibalistic spider. Ethology 122:257-266. DOI: 10.1111/eth.12466

Schulte KF, Uhl G, and Schneider JM. 2010. Mate choice in males with one-shot genitalia: limited importance of female fecundity. Animal Behaviour 80:699-706. 10.1016/j.anbehav.2010.07.005

Schulz S. 2013. Spider pheromones - a structural perspective. Journal of Chemical Ecology 39:1-14. 10.1007/s10886-012-0231-6

Schwartz SK, Wagner WE, and Hebets EA. 2013. Spontaneous male death and monogyny in the dark fishing spider. Biology Letters 9. 10.1098/rsbl.2013.0113

Sparkes TC, Keogh DP, and Pary RA. 1996. Energetic costs of mate guarding behavior in male streamdwelling isopods. Oecologia 106:166-171. 10.1007/bf00328595

Stearns SC. 2000. Life history evolution: successes, limitations, and prospects. Naturwissenschaften 87:476-486. 10.1007/s001140050763 
674 Stoffer B, and Uetz GW. 2015. The effects of social experience with varying male availability on female 675 mate preferences in a wolf spider. Behavioral Ecology and Sociobiology 69:927-937.

$676 \quad 10.1007 / \mathrm{s} 00265-015-1904-2$

677 Stoffer B, and Uetz GW. 2016. Social experience affects female mate preferences for a visual trait in a 678 wolf spider. Behavioral Ecology 27:252-261. 10.1093/beheco/arv143

679 Stoltz JA, McNeil JN, and Andrade MCB. 2007. Males assess chemical signals to discriminate just-mated 680 females from virgins in redback spiders. Animal Behaviour 74:1669-1674.

$681 \quad$ 10.1016/j.anbehav.2007.03.011

682 Therneau T. 2014. _A Package for Survival Analysis in S_. Available at R package version 2.37-7, $683 \quad<U R L:$ http://CRAN.R-project.org/package $=$ survival $>$.

684 Thomas ML. 2011. Detection of female mating status using chemical signals and cues. Biological 685 Reviews 86:1-14. 10.1111/j.1469-185X.2010.00130.x

686 Trivers RL. 1972. Parental investment and sexual selection. Sexual selection and the descent of man 687 Aldine Publishing Co., 136-179.

688 Tuni C, and Berger-Tal R. 2012. Male preference and female cues: males assess female sexual maturity 689 and mating status in a web-building spider. Behavioral Ecology 23:582-587.

$690 \quad 10.1093 /$ beheco/ars001

691 Uhl G, Nessler SH, and Schneider J. 2007. Copulatory mechanism in a sexually cannibalistic spider with 692 genital mutilation (Araneae : Araneidae : Argiope bruennichi). Zoology 110:398-408.

$693 \quad 10.1016 /$ j.zool.2007.07.003

694 Uhl G, Nessler SH, and Schneider JM. 2010. Securing paternity in spiders? A review on occurrence and 695 effects of mating plugs and male genital mutilation. Genetica 138:75-104. 10.1007/s10709-009$696 \quad 9388-5$

697 Uhl G, Zimmer SM, Renner D, and Schneider JM. 2015. Exploiting a moment of weakness: male spiders 698 escape sexual cannibalism by copulating with moulting females. Scientific Reports 5. $699 \quad 10.1038 /$ srep16928

700 Vahed K. 1998. The function of nuptial feeding in insects: review of empirical studies. Biol Rev Camb $701 \quad$ Philos Soc 73:43-78. 10.1017/s0006323197005112

702 Watson PJ. 1986. Transmission of a female sex pheromone thwarted by males in the spider Linyphia $703 \quad$ litigiosa (Linyphiidae). Science 233:219-221. 10.1126/science.3726530

704 Wedell N, Gage MJG, and Parker GA. 2002. Sperm competition, male prudence and sperm-limited 705 females. Trends in Ecology \& Evolution 17:313-320. 10.1016/s0169-5347(02)02533-8

706 Weir LK, Grant JWA, and Hutchings JA. 2011. The influence of operational sex ratio on the intensity of 707 competition for mates. American Naturalist 177:167-176. 10.1086/657918 
708 Welke KW, and Schneider JM. 2010. Males of the orb-web spider Argiope bruennichi sacrifice themselves to unrelated females. Biology Letters 6:585-588. 10.1098/rsbl.2010.0214

710

711

712

713

714

715

716

717

718

719

720

721

722

723

724

725

Welke KW, and Schneider JM. 2012. Sexual cannibalism benefits offspring survival. Animal Behaviour 83:201-207. 10.1016/j.anbehav.2011.10.027

Welke KW, Zimmer SM, and Schneider JM. 2012. Conditional monogyny: female quality predicts male faithfulness. Frontiers in Zoology 9. 10.1186/1742-9994-9-7

Wilder SM, and Rypstra AL. 2008. Sexual size dimorphism predicts the frequency of sexual cannibalism within and among species of spiders. American Naturalist 172:431-440. 10.1086/589518

Wilder SM, Rypstra AL, and Elgar MA. 2009. The importance of ecological and phylogenetic conditions for the occurrence and frequency of sexual cannibalism. Annual Review of Ecology Evolution and Systematics, 21-39.

Wyatt TD. 2014. Pheromones and animal behavior: chemical signals and signatures, 2nd Edition. New York: Cambridge University Press.

Zimmer SM, and Schneider JM. 2016. Fine-scale spatial genetic structure suggests modest risk of inbreeding in natural populations of Argiope bruennichi. Evolutionary Ecology Research 17:3551.

Zimmer SM, Welke KW, and Schneider JM. 2012. Determinants of natural mating success in the cannibalistic orb-web spider Argiope bruennichi. Plos One 7. 10.1371/journal.pone.0031389 
Figure 1

Scheme of experimental set-up.

Males were placed in test arenas $(100 \times 70 \times 50 \mathrm{~cm})$ and exposed either to $(A)$ four virgin adult females (high mate availability) or to (B) one adult, virgin female and three penultimate females (low mate availability). Adult virgin females are black and penultimate females greycolored.

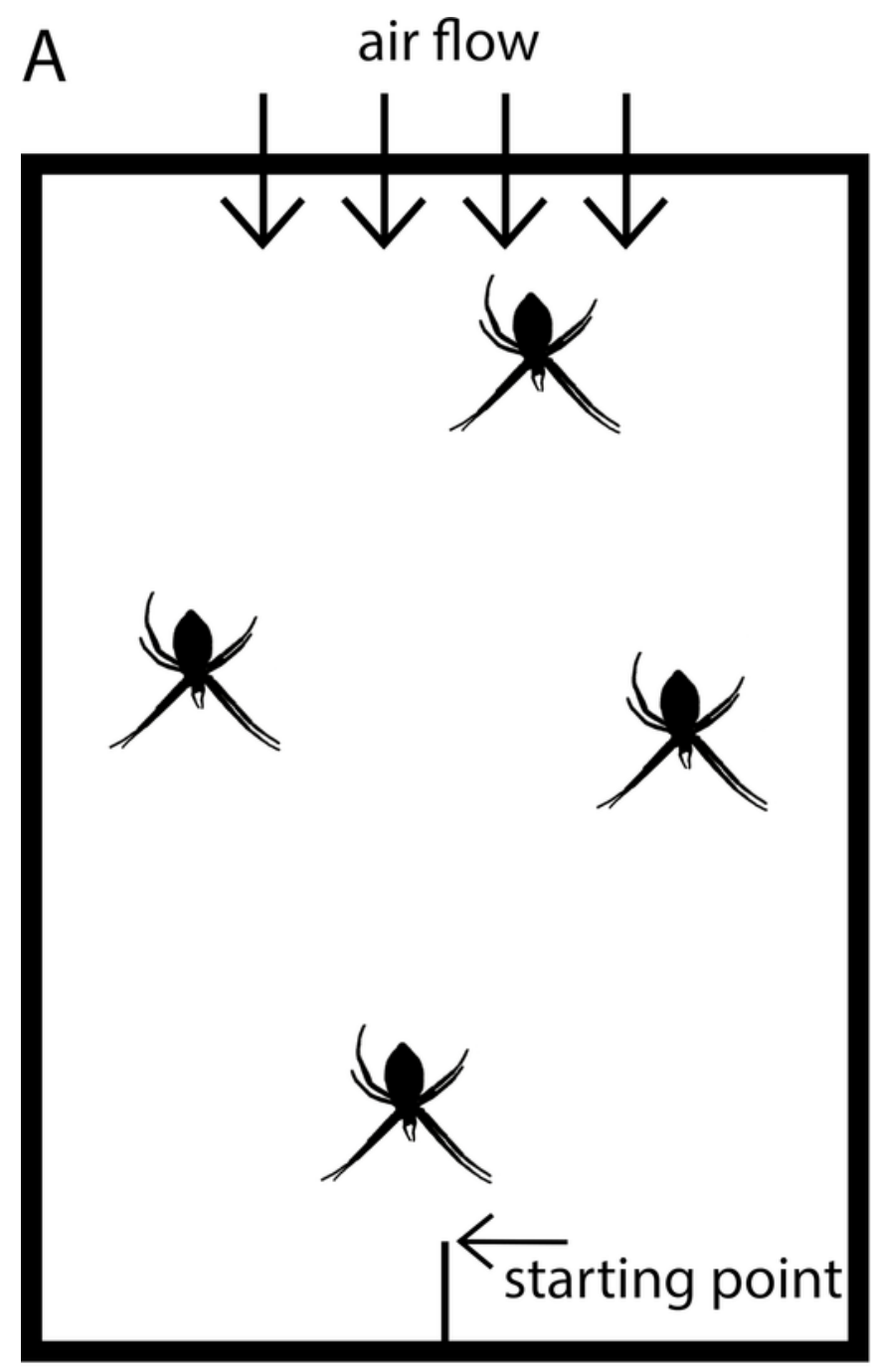

high mate availability

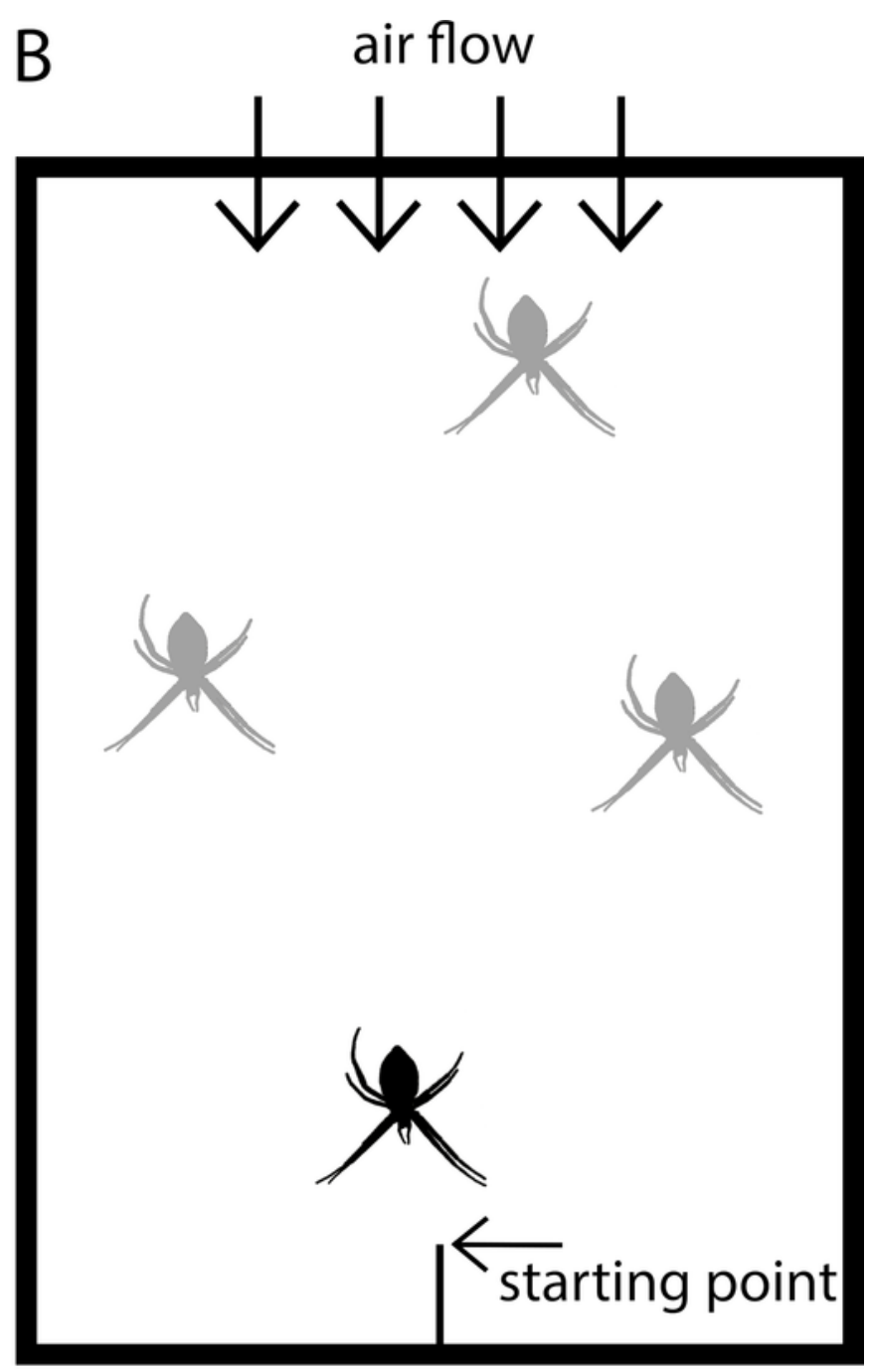

low mate availability 
Figure 2 (on next page)

Effects of mate availability on mating behaviour.

Effects of mate availability (high, low) on (A) the copulation duration (Cox regression: $\mathrm{N}=50$, $\mathrm{Chi}^{2}=3.6061 \mathrm{p}=0.0576$ ); (B) the relative frequency of sexual cannibalism (Logistic regression: $\mathrm{N}=50$, df-deviance $=0.3082, \mathrm{p}=0.5788$ ); and $(\mathrm{C})$ the relative frequency of males that survived the first copulation and copulated a $2^{\text {nd }}$ time with the same female (monopolisation) (Fisher exact test: $N=19, p=0.1698$ ). 

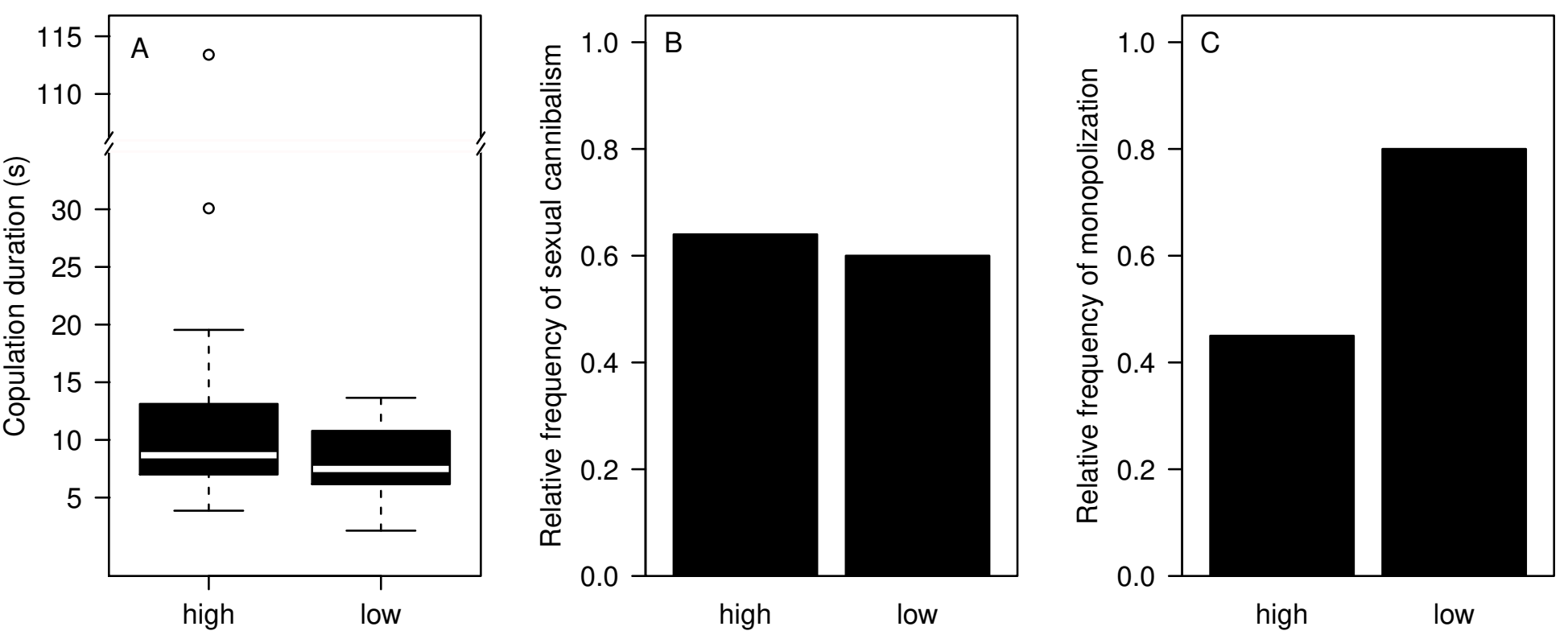

Mate availability 


\section{Figure 3 (on next page)}

Male size effect on sexual cannibalism.

Males that were cannibalized by the female during their first copulation were larger in body

size than males that escaped the female attack (logistic regression: $N=50$, df-deviance $=4.97$, $\mathrm{p}=0.0258)$. 


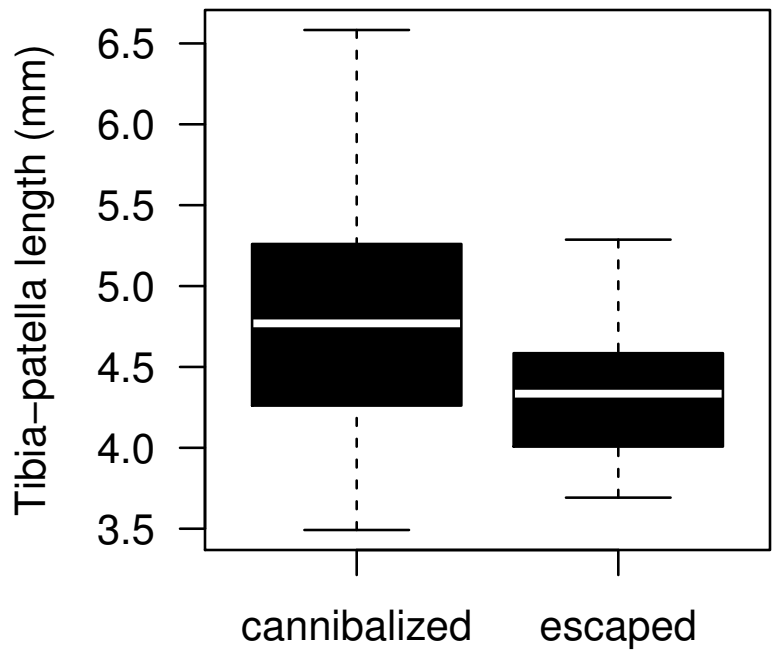

Outcome of 1 . copulation for males 


\section{Table $\mathbf{1}$ (on next page)}

Results of the Cox regression model testing effects of mate availability (low, high) on the copulation duration $(\mathrm{N}=50)$.

The effect of male size was tested in a different model because the sample size was reduced $(\mathrm{N}=46)$. The coefficients (coef) and their standard errors (se), and the hazard ratios (exp(coef)) are taken from the full model. 


\begin{tabular}{|c|c|c|c|c|c|}
\hline Effect & coef \pm se & $\exp (\operatorname{coef})$ & $\mathrm{Chi}^{2}$ & df & $\mathbf{p}$ \\
\hline Availability of virgin $q$ q(low) & $0.5743 \pm 0.3166$ & 1.7759 & 3.6061 & 1 & 0.0576 \\
\hline q size & $0.0703 \pm 0.1177$ & 1.0728 & 0.3567 & 1 & 0.5503 \\
\hline O condition & $0.0052 \pm 0.0026$ & 1.0052 & 3.0991 & 1 & 0.0783 \\
\hline$\delta$ relative weight change & $-2.0859 \pm 1.6427$ & 0.1242 & 1.4882 & 1 & 0.2225 \\
\hline Effect & coef \pm se & $\exp (\operatorname{coef})$ & $\mathrm{Chi}^{2}$ & df & $\mathbf{p}$ \\
\hline$\hat{o}$ size & $0.1032 \pm 0.2499$ & 1.1087 & 0.1683 & 1 & 0.6816 \\
\hline
\end{tabular}

1 


\section{Table 2 (on next page)}

Results of the binary logistic regression testing effects of mate availability (low, high) on the cannibalism rate $(\mathrm{N}=50)$.

The effect of male size was tested in a different model because the sample size was reduced $(\mathrm{N}=46)$. The estimates and standard errors (SE) of the estimates are logit-transformed. Brackets show estimates and standard error of the full model and results of the minimal adequate model are without brackets. 


\begin{tabular}{|c|c|c|c|c|c|}
\hline Effect & & Estimates \pm SE & df-deviance & df & $\mathbf{p}$ \\
\hline \multirow[t]{2}{*}{ Intercept } & \multirow{2}{*}{$\begin{array}{l}\text { (Reference level: } \\
\text { high availability) }\end{array}$} & $(-3.0123 \pm 2.2115)$ & & & \\
\hline & & $0.4895 \pm 0.2914$ & & & \\
\hline Availability of virgin $q$ 우 & (low availability) & $(-0.3185 \pm 0.6160)$ & 0.3082 & 1 & 0.5788 \\
\hline o size & & $(0.4500 \pm 0.2861)$ & 2.2874 & 1 & 0.1304 \\
\hline q condition & & $(-0.0023 \pm 0.0056)$ & 0.1676 & 1 & 0.6822 \\
\hline$\delta$ relative weight change & & $(-2.6694 \pm 3.8577)$. & 0.3953 & 1 & 0.5295 \\
\hline Effect & & Estimates \pm SE & df-deviance & df & $\mathbf{p}$ \\
\hline Intercept & & $-5.0451 \pm 2.7963$ & & & \\
\hline ô size & & $1.2490 \pm 0.6213$ & 4.9700 & 1 & 0.0258 \\
\hline
\end{tabular}

1 